\title{
TRABAJO COLABORATIVO INTERDISCIPLINARIO PARA LA PROMOCIÓN DE LA EDUCACIÓN INCLUSIVA Y EL ÉXITO ESCOLAR DE ESTUDIANTES DE PRIMARIA EN COSTA RICA Collaborative Interdisciplinary Work for the Promotion of Inclusive Education and School Success of Students in Costa Rican Primary Schools
}

Irma Arguedas-Negrini ${ }^{1}$

irma.arguedas@ucr.ac.cr

María de los Ángeles Carpio-Brenes ${ }^{1}$ maria.carpiobrenes@ucr.ac.cr

${ }^{1}$ Universidad de Costa Rica. Costa Rica

Recibido: $28 / 01 / 2021$

Revisado: $28 / 04 / 2021$

Aprobado: $12 / 05 / 2021$

Preprint: 25/06/2021

Publicado: 15/07/2021

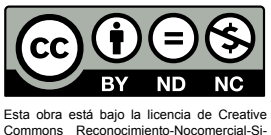

\section{Resumen}

En el desarrollo integral de las personas y los países, el éxito escolar de niñas y niños, en el marco de la educación inclusiva, es esencial; uno de los componentes para el logro del mismo es el trabajo colaborativo, por lo que se consideró pertinente indagar acerca de las acciones interdisciplinarias para la promoción del éxito escolar a nivel de primaria. El presente artículo divulga los hallazgos de corte cualitativo de una investigación que analizó el trabajo colaborativo de profesionales en Orientación, docentes de Educación Especial y profesorado de Educación General Básica en escuelas costarricenses. La información se recogió mediante la aplicación de entrevistas semiestructuradas a 17 profesionales. Se presenta una definición de éxito escolar construida a partir de los aportes de las personas participantes, se describe la multiplicidad de acciones colaborativas que llevan a cabo en función del éxito escolar de sus estudiantes y se mencionan los retos a los que se enfrentan diariamente, algunos de los cuales son factores externos a la institución educativa. Se concluye que tanto el éxito escolar como el trabajo colaborativo son concebidos por las personas informantes como procesos que se construyen en conjunto y que requieren, además de las acciones, ser sustentados en convicciones acerca del derecho a la educación, la integralidad del desarrollo durante la nińez y las actitudes de respeto y empatía.

Palabras clave: ambiente educacional, educación especial, educación inclusiva, estudiante de primaria, éxito escolar, orientación escolar, trabajo colaborativo interdisciplinario.

\section{Abstract}

In the holistic development of individuals and countries, the school success of girls and boys, is essential within the framework of inclusive education; one of the components for its achievement is collaborative work; therefore, it was considered pertinent to inquire about interdisciplinary actions for the promotion of school success at the primary school level. This article reports the qualitative findings of an investigation that analyzed the collaborative work of Orientation professionals, Special Education teachers and General Basic Education teachers in Costa Rican schools. The information was collected through semi-structured interviews with 17 professionals. A definition of school success is presented based on the contributions of the participants, describing the multiplicity of collaborative actions they carry out for the school success of their students and mentioning the challenges they face on a daily basis, some of which are external factors to the educational institution. It is concluded that both school success and collaborative work are conceived by the informants as processes that are built together and that require, in addition to actions, to be supported by convictions about the right to education, the holistic development during childhood and attitudes of respect and empathy.

Keywords: educational environment, educational guidance, inclusive education, interdisciplinary collaborative work, primary school student, school success, special education. 


\section{Introducción}

La promoción del éxito escolar, comprendido como el desarrollo integral de cada estudiante, es una tarea compartida entre la totalidad de actores de la comunidad educativa y tiene efectos en el bienestar de las personas a lo largo de todo el ciclo vital. A partir de la emisión, por parte del Ministerio de Educación Pública de Costa Rica (MEP), de las Líneas de acción para los Servicios de Apoyo Educativo que se brindan desde la Educación Especial (MEP, 2018), así como de los nuevos programas de estudio de Orientación y la transformación curricular centrada en una nueva ciudadanía (MEP, 2017b), se llevó a cabo una investigación que tuvo el objetivo de analizar las prácticas de trabajo colaborativo que implementan profesionales de Orientación y Educación Especial, junto con el profesorado de Educación Primaria, para la promoción del éxito escolar y la permanente construcción de instituciones inclusivas.

La presente investigación se planteó en respuesta al Programa Estado de la Nación, el cual reportó en su Sexto informe del estado de la educación (PEN, 2017) bajos desempeńos en Primaria, sobre todo al pasar al tercer ciclo, y un estancamiento del logro en Secundaria en el estudiantado costarricense. En dicho informe se recomendó volver la mirada hacia las escuelas, así como conocer más a fondo lo que sucede en las aulas.

El MEP no ha sido indiferente ante la problemática del bajo desempeño, y ha hecho grandes esfuerzos por prevenir la exclusión educativa y promover la inclusión mediante la eliminación de barreras que dificultan el éxito integral de la totalidad del estudiantado (MEP, 2017b; MEP, 2018).

La educación inclusiva es un proceso continuo de transformación de los valores y cultura escolar porque representa un cambio de una perspectiva centrada en el déficit a una que promueve el éxito; requiere, sin embargo, apoyos y estrategias para su implementación, por lo que es recomendable comenzar por conocer las prácticas educativas, como medida para garantizar la calidad de este proceso (Schuelka, 2018).
En el marco de la educación inclusiva, el trabajo colaborativo es la clave para coordinar acciones conjuntas entre profesionales de diferentes disciplinas. Esta herramienta "tiene como finalidad alcanzar objetivos comunes y consensuados, desde la toma de decisiones, el compartir responsabilidades y aportar conocimientos, prácticas y valores, desde los diferentes saberes de cada persona" (MEP, 2018, p. 26). El diseńo universal para el aprendizaje (DUA) es otra herramienta esencial de la educación inclusiva (MEP, 2018), por lo que el estudio se focalizó en el trabajo colaborativo y la implementación conjunta del DUA.

Se utilizó una metodología cualitativa. La técnica de recolección de información fue la entrevista semiestructurada, para cuya aplicación se siguió una guía (Anexo 1). En este artículo se presentan los hallazgos obtenidos: se encontró que las personas participantes ven el éxito escolar como un proceso integral que se construye en conjunto; asimismo, se obtuvo la descripción de múltiples acciones colaborativas y de los retos que enfrentan los equipos en las escuelas de educación primaria participantes.

\section{Revisión de la literatura}

\subsection{Educación inclusiva}

La educación inclusiva se enfoca en eliminar los obstáculos presentes en los contextos en que se desenvuelven las personas más que en las dificultades que estas presentan. Es por eso que desde un enfoque inclusivo se valora la diversidad y se promueve una participación equitativa en la sociedad mediante la eliminación de las barreras que colocan en condición de riesgo de exclusión al estudiantado más vulnerable (Coronado-Hijón, 2018).

La educación inclusiva favorece la justicia social porque busca ofrecer las condiciones para el éxito de todo el estudiantado. De hecho, se encuentra dentro de los propósitos establecidos por la Organización de las Naciones Unidas en la Agenda 2030 (Arnaiz et al., 2018). La educación inclusiva se posiciona como un paradigma que no acepta un único perfil 
de estudiante, sino una gran diversificación, cuya responsabilidad no es exclusiva de las niñas, los nińos y sus familias, sino que se comparte con toda la comunidad educativa; es la base de una sociedad que ofrece dignidad a todos sus integrantes en contraposición a las prácticas excluyentes, y permite responder a la función esencial de la educación, a saber, un escenario rico en oportunidades para el desarrollo del estudiantado en diferentes ámbitos (Neto et al., 2018).

A nivel de cada institución, la educación inclusiva implica que la atención a la diversidad sea una de las tareas fundamentales, lo cual, como se anotó, requiere estrategias que permitan identificar y atender tempranamente al estudiantado vulnerable mediante un abordaje que tome en cuenta el conjunto de factores que intervienen en el éxito escolar, a saber, "los del contexto (de las políticas), los escolares (que incluyen desde el proyecto de centro hasta las relaciones entre los actores), los relativos al aula (donde resultan cruciales los materiales educativos y las estrategias de enseńanza), y los del alumnado" (Aristimuño \& Parodi, 2017, p. 142). Asimismo, el PEN (2017) considera urgente el abordaje educativo que toma en cuenta la diversidad y las necesidades específicas del estudiantado.

Para ello es indispensable la articulación de acciones de forma interdisciplinaria, para el análisis y resolución de los problemas que inciden en el quehacer de las instituciones educativas. De esta forma es que "lo interdisciplinario aparece, cuando la distribución de las funciones y la importancia de cada saber se definen en relación al problema y no por el peso o la tradición de cada profesión" (Mondino, 2017, p. 69). Se da una práctica compartida, enfocada en la prevención y en la atención de situaciones problemáticas del ámbito escolar que deben ser abordadas mediante espacios de discusión y reflexión en el interior de equipos de trabajo conformados por profesionales involucrados en el proceso educativo (Bertoldi \& Enrico, 2012). Si bien se trata de reestructuraciones que generan variados grados de incertidumbre, estas han demostrado ser beneficiosas para la totalidad de estudiantes (Schuelka, 2018).
Para el MEP, "la educación inclusiva es la estrategia, los apoyos, el diseño universal para el aprendizaje y el trabajo colaborativo son algunas de las herramientas que nos permitirán avanzar hacia una educación para la diversidad en nuestros centros educativos" (MEP, 2018, p. 6).

Los apoyos son aquellos recursos que buscan promover el desarrollo, la educación, los intereses y el bienestar de una persona y que mejoran el funcionamiento individual: pueden brindarse de manera temporal o permanente y se clasifican en cuatro tipos: personales, materiales o recursos tecnológicos, organizativos y curriculares (MEP, 2013).

Los apoyos personales son los que requieren la participación de otras personas distintas al personal docente de aula, como, por ejemplo, las personas profesionales en Orientación, en Educación Especial, en Psicología, Terapia Física, Terapia de Lenguaje, compañeras y compañeros de aula, familias y otras involucradas en el proceso educativo. Los materiales o recursos tecnológicos son los medios que facilitan el acceso del estudiantado a las experiencias del currículo escolar. Los apoyos organizativos están relacionados con el agrupamiento del estudiantado, así como la distribución del tiempo, los espacios y el clima organizacional del aula. Cuando se trata de ajustes, adaptaciones o modificaciones temporales o permanentes de los elementos del currículo en los programas de estudio para responder a las necesidades específicas del estudiantado, se denominan apoyos curriculares (MEP, 2013).

\subsection{Trabajo colaborativo interdisciplinario}

Dos disciplinas que intervienen al lado de la Educación General Básica en la promoción del éxito escolar son la Orientación y la Educación Especial. Dentro de las funciones de profesionales de Orientación en la Educación Primaria en Costa Rica está, de acuerdo con el MEP (2017a, p. 7), la coordinación con el personal del centro para la "inclusión y promoción académica que favorezcan la permanencia y el éxito escolar", así como la implementación de "estrategias para la atención, valoración, referencia y seguimiento 
de estudiantes que presenten vulnerabilidad de índole académica, emocional y socioeconómica”.

Como promotora del desarrollo académico, vocacional y personal-social del estudiantado, la Orientación en la escuela primaria promueve la igualdad en el acceso a las oportunidades y la participación de todo el estudiantado. La persona profesional en Orientación está llamada a favorecer, junto con otras disciplinas y actores, un clima institucional de apoyo para el logro y la inclusión. Se aspira a que las instituciones educativas se caractericen por lo que bien resumen Marshak et al. (2010): la totalidad de estudiantes sienten que pertenecen y se les transmite valoración, la responsabilidad por el logro del estudiantado es compartida, y hay eliminación de discriminación y de barreras para una inclusión genuina, que no es la mera presencia, sino la participación plena en oportunidades educativas.

El personal de orientación favorece la adquisición de habilidades para el estudio, la toma de decisiones y la organización, y ayuda a cada estudiante a comprender sus necesidades particulares de aprendizaje, sus fortalezas y otros recursos para enfrentar desafíos (Buckley \& Mahdavi, 2018). Estas autoras agregan que, siendo las habilidades sociales un factor protector para el estudiantado en general y para quienes tienen discapacidad $\mathrm{u}$ otras condiciones en particular, es crucial que se atiendan las necesidades de desarrollo en este ámbito.

Además, la Orientación contribuye con la construcción de ambientes de apoyo que brindan a nińas y niños la aceptación que les permite explorar sus sentimientos, inseguridades y retos, con miras a la implementación de soluciones. Lo opuesto a un clima escolar de apoyo y confianza es el que presenta muchas experiencias de aprendizaje frustrantes, poca valoración del esfuerzo y habilidades del estudiantado, con la consiguiente limitación de la expresión de las potencialidades.

Todo lo anterior guarda concordancia con el MEP (2017b) y su concepción de Educar para una nueva ciudadanía. En los nuevos programas de estudios de Orientación se expone que la nueva ciudadanía pretende el abordaje preventivo de situaciones como la salida anticipada del sistema educativo, la inclusión social, el balance entre los intereses individuales y los de la colectividad, el desarrollo de la criticidad, la reducción de las barreras en la enseńanza y el conocimiento de las oportunidades que ofrece el medio. Los programas parten del derecho del estudiantado a una educación integral y respetuosa de la diversidad. Se expone la incidencia de la Orientación en la permanencia y el éxito escolar al promover la potenciación de conocimientos, habilidades, actitudes y valores para enfrentar retos en los ámbitos educativo, vocacional y personal-social. Para conseguir estos propósitos, la persona profesional interviene tanto de forma directa con el estudiantado, como indirecta con el trabajo con familias, otro personal y la institución como un todo.

Por su parte, la persona profesional en Educación Especial está llamada a "prestar el apoyo necesario para que, en el marco del sistema general de educación, se haga efectivo el derecho del estudiantado con discapacidad a la educación inclusiva, según lo demanda la Convención sobre los derechos de las personas con discapacidad" (MEP, 2018, p. 22). Esto implica que debe dejar de lado las antiguas prácticas de atención individualizada fuera del contexto de aula para "acompañar tanto al estudiantado con discapacidad, sus compañeros y compañeras de aula, sus colegas docentes, como a las familias y otros profesionales y miembros de la comunidad involucrados en el proceso educativo" (MEP, 2018, p. 25).

El apoyo necesario para que se haga efectivo el derecho a la educación inclusiva se aplica a la totalidad del estudiantado, independientemente de la condición física, sensorial, mental o intelectual. De acuerdo con Arnaiz (2012), el propósito medular de los centros educativos del siglo XXI es "promover una educación democrática e inclusiva que garantice el derecho de todos los nińos y jóvenes a recibir una educación de calidad basada en los principios de igualdad, equidad y justicia social" (p. 26). La autora agrega, por consiguiente, que la escuela debe ser accesible a todas las personas en condiciones adecuadas para sus características y necesidades, es decir, respondiendo 
a la diversidad es como se garantiza la equidad, indispensable para la cohesión social. Si no hay respuestas educativas a las necesidades del alumnado más vulnerable, se amenazan sus derechos al dejarle en riesgo de exclusión, desigualdad y marginación.

Le corresponde al profesorado de Educación Especial garantizar la atención a la diversidad, evitando cualquier tipo de barrera en el aprendizaje y la participación, sin limitarse, como señala Coronado-Hijón (2018), a las necesidades educativas específicas, sino que ha de contemplar los obstáculos que el estudiantado enfrenta al interactuar y participar en el contexto educativo. La educación inclusiva atribuye las dificultades del estudiantado no a un déficit personal, sino a las barreras que se presentan, tanto por falta de recursos o de experiencia como por la existencia de programas, de métodos de enseñanza o de actitudes inadecuadas que pueden limitar la participación y el aprendizaje.

En el contexto educativo costarricense, a partir de febrero del año 2018 el MEP puso en vigencia el documento denominado Líneas de acción para los Servicios de Apoyo Educativo que se brindan desde la Educación Especial en Educación Preescolar y en Primero y Segundo Ciclo de la Educación General Básica, en el que establece cuatro principios orientadores para el profesorado de Educación Especial en su calidad de docentes de apoyo educativo:

a) El respeto a la dignidad inherente a toda persona, enfocado en la autonomía, sin juicios de ninguna índole, respetando las diferencias culturales, de género, orientación sexual, condición de discapacidad, religión y estilos de aprendizaje.

b) La igualdad y la equidad de oportunidades, es decir, dar las mismas oportunidades a todas y todos y brindarles oportunidades, considerando las diferentes características de cada estudiante.

c) La participación, en la que se debe garantizar la individualidad de cada persona y asegurarse de que su voz sea escuchada.

d) El trabajo colaborativo, con el fin de alcanzar objetivos comunes y consensuados; todo para el mejoramiento continuo del proceso educativo (MEP, 2018).

En el marco de la educación inclusiva, el trabajo colaborativo es la clave para coordinar acciones conjuntas entre profesionales de diferentes disciplinas. $\mathrm{Su}$ finalidad es el logro de propósitos consensuados, la responsabilidad compartida y la valoración de los saberes cognitivos y prácticos de cada integrante (MEP, 2018).

Esto lo reafirma Arnaiz (2012), quien plantea que, para construir inclusión y eficacia en las escuelas, se requiere la colaboración. De acuerdo con la autora, sin colaboración interprofesional no es posible una escuela inclusiva; agrega que en su implementación deben evitarse las relaciones verticales y más bien favorecer la igualdad y los principios compartidos. Esta posición se asemeja a la de Barrow y Mamlin (2016), los cuales consideran que la colaboración requiere confianza mutua, respeto y apertura. Para el caso específico de profesionales en Orientación y docentes de Educación Especial, exponen que la colaboración es particularmente relevante en los esfuerzos por alcanzar el logro escolar y el desarrollo integral de la totalidad del estudiantado.

Este trabajo colaborativo interdisciplinario se ve enriquecido por el soporte decidido de las direcciones de las instituciones y por un clima de respeto y aceptación. Asimismo, son necesarias la apertura al cambio y la reflexión acerca de prácticas habituales (Arnaiz et al., 2018). Con respecto al DUA, surge del diseño universal del ámbito arquitectónico, que se refiere al "diseño de productos y entornos que cualquier persona pueda utilizar, en la mayor medida posible, sin necesidad de una adaptación posterior destinada a un público específico" (Centro para la Tecnología Especial Aplicada (CAST), citado por Alba et al., 2011, p. 5).

\subsection{Diseño universal para el éxito escolar}

El DUA es una herramienta que favorece el éxito escolar en el estudiantado en general, entendido este no solo como la obtención de altas calificaciones sino también como la adquisición de habilidades 
que conducen al desarrollo personal y social de las personas (Confederación Española de Asociaciones de Padres y Madres de Alumnos, 2011).

Con el DUA como herramienta para la inclusión, se espera atender a la diversidad mediante la flexibilidad curricular, cumpliendo los siguientes tres principios fundamentales en la programación de aula (Alba et. al, 2011):

Principio 1: Múltiples medios de representación. Cada estudiante es diferente en la forma en que percibe y comprende la información que se le presenta, por lo que, al diseñar las actividades de clase, es necesario considerar: a) las diferentes opciones de percibir la información (auditiva, visual, táctil), brindando material para los diferentes canales sensoriales; b) las distintas opciones para el lenguaje y los símbolos, ajustando los sistemas de representación de la información, lingüísticos o no, a las variaciones de acuerdo con las realidades culturales y sociales del estudiantado; c) las diversas alternativas para la comprensión, mediante diferentes procesos de enseñanza-aprendizaje que permitan la transmisión de información para la comprensión de la misma.

Principio 2: Múltiples medios de expresión. Debido a que el estudiantado tiene diferentes formas de aprender y de manifestar ese conocimiento, es necesario proporcionar diferentes: a) medios físicos de acción, lo que implica que los materiales que se utilicen en la enseñanza tengan un diseño sencillo; b) opciones para la expresión, que incluyen acciones como el utilizar múltiples formas o medios de comunicación, usar variedad de herramientas para la composición y la construcción e incorporar niveles graduados de apoyo en los procesos de aprendizaje; c) acciones para las funciones ejecutivas, lo que implica actividades como guiar el establecimiento de metas adecuadas, apoyar la planificación y el desarrollo de estrategias, facilitar la gestión de información y recursos, así como mejorar la capacidad para hacer un seguimiento de los avances.

Principio 3: Múltiples medios de motivación. Este se refiere al elemento afectivo, el cual es crucial en los procesos de aprendizaje y se evidencia en las formas en que el estudiantado se implica en su proceso educativo (Alba et al., 2011). En consecuencia, hay que plantear alternativas para:

a) captar el interés, es decir, lograr la atención y el involucramiento del estudiantado al optimizar la elección individual y la autonomía, la relevancia, el valor y la autenticidad, minimizar la sensación de inseguridad y las distracciones;

b) mantener el esfuerzo y la persistencia mediante el desarrollo de habilidades que le permitan al estudiantado realizar un equilibrio entre aspectos de atención, motivación y esfuerzo-persistencia;

c) la autorregulación, proporcionando las herramientas necesarias para llevar a cabo un adecuado proceso de regulación de las propias emociones y motivaciones.

Taylor (1989) y otros autores como Nelsen et al. (2016) enfatizan en que la mejor forma de motivar el trabajo escolar y el buen comportamiento es la satisfacción de la necesidad de sentirse una persona valiosa, lo cual se logra ofreciendo posibilidades de elegir y promoviendo un ambiente seguro, es decir, en el que se validan los sentimientos de las nińas y los nińos, lo que no significa aceptar comportamientos irrespetuosos. Tampoco significa que la persona adulta pierda su autoridad, se evita tanto la dominación como la sobreprotección. Igualmente, se requiere un equilibrio en relación con las expectativas, las cuales deben ser realistas y no demasiado bajas ni demasiado altas para las posibilidades del alumno o la alumna en concreto. Al respecto, Ulloa (2017) propone:

Propiciar que, en el trabajo en clase, la forma como el estudiantado percibe las características de las tareas se traduzca en una percepción de que se cuenta con excelentes oportunidades para efectuarlas exitosamente, y que se conviertan así en causas positivas para un buen desempeño estudiantil (p. 207).

El comportamiento autorregulado, que tanto interesa en el centro educativo, solo es posible si la persona se siente segura y motivada, de forma tal que puede adaptar sus cogniciones y estados afectivos a 
los requerimientos de sus vivencias escolares. Una acción relevante de las personas adultas es contribuir con la identificación de emociones y cogniciones y con el desarrollo de las habilidades para modificarlas, si es que resultan contraproducentes (Ulloa, 2017).

\section{Método}

\subsection{Tipo de investigación}

Debido a que se consideraba necesario profundizar en las perspectivas de las personas participantes acerca de sus experiencias con el trabajo colaborativo y los aportes desde las respectivas disciplinas al llevarlo a cabo, se utilizó un enfoque cualitativo. Se eligió el método fenomenológico porque pone el énfasis en las experiencias de los seres humanos y su relación con sucesos, objetos y otras personas de su contexto (Álvarez-Gayou, 2003). El método fenomenológico permite recoger las perspectivas de las personas participantes acerca del fenómeno de interés (Gill, 2020).

\subsection{Población participante}

Tal y como lo expone Castillo (2020), en un estudio fenomenológico la información proviene de quienes han experimentado el fenómeno en estudio. Es por esto que las personas que se invitaron a participar se seleccionaron mediante un muestreo no probabilístico por conveniencia en escuelas primarias que contaran con: profesional en Orientación, al menos una persona de apoyo educativo en Educación Especial, una persona docente de primer ciclo y una de segundo ciclo con quien ambos profesionales colaboraran. Participaron cuatro escuelas, con un número inicial de 16 personas; no obstante, en una de las instituciones la directora manifestó su deseo de ser incluida, por lo que el número de participantes se amplió a 17.

\subsection{Técnica para la recolección de información cualitativa}

Con el fin de obtener las perspectivas de las personas participantes acerca de las experiencias de trabajo colaborativo, al recoger información era necesario que reflexionaran acerca del fenómeno de estudio y lo pudieran describir en detalle, y se procedió a hacer entrevistas semiestructuradas. De acuerdo con MacQueen (2007), en este tipo de entrevistas el tema y el contenido son definidos por la persona investigadora, pero la formulación y el orden de las preguntas pueden variar.

Con base en el referente teórico, se elaboró una guía de entrevista, la cual se sometió a validación con personas expertas con formación en Educación Primaria, Problemas de aprendizaje, Educación Especial, Educación Secundaria, Orientación y Evaluación Educativa, así como con experiencia en trabajo colaborativo. La validación consistió en la emisión de criterios con respecto a la comprensibilidad de las preguntas y a la relación con los objetivos de la investigación.

Las preguntas se diseñaron para indagar las perspectivas acerca del éxito escolar y de las acciones colaborativas que implementan para su logro. Antes de realizar las entrevistas, cada participante leyó y firmó el consentimiento informado. Para efectos de la presentación de resultados, se asignaron códigos de P1 a P17 en sustitución de los nombres de las personas participantes.

\subsection{Procedimiento para el análisis de la información}

El cuerpo de información cualitativa está constituido por las transcripciones de las 17 entrevistas semiestructuradas. Inicialmente, estas fueron leídas repetidamente por separado, para luego pasar a la puesta en común entre investigadoras. En la siguiente fase cada investigadora procedió a asignar códigos al cuerpo de información y se señalaron posibles citas textuales a utilizar para explicar los hallazgos. Se utilizó la herramienta Atlas.ti, versión 8.

Posteriormente se hizo una revisión adicional para asegurarse de que todos los extractos de información que se habían codificado correspondían a las categorías identificadas. La identificación y definición de códigos definitivos se hizo por consenso. Entonces se procedió a identificar patrones que permitieran agrupar los códigos para establecer categorías que 
reflejaran las perspectivas de las personas participantes con respecto a los interrogantes del estudio; esto también se hizo por consenso, con triangulación entre investigadoras.

Cabe agregar que hubo bastante acuerdo entre las perspectivas de las personas participantes. Cuando el análisis no evidenció nuevas ideas o conceptos, es decir, cuando se presentó redundancia, se consideró que se había alcanzado la saturación de la información (Bowen, 2008), lo que sucedió antes de concluir con la codificación de la totalidad de entrevistas; no obstante, se continuó para de esa forma incluir las perspectivas de las 17 personas.

Como se indicó, la indagación giró alrededor de las acciones que las personas participantes realizan en conjunto en función del éxito escolar de la totalidad de sus estudiantes de primaria. De la información recogida se desprendieron múltiples acciones, e inicialmente una de las categorías se denominó "Acciones colaborativas"; no obstante, al irse desplegando la presencia de una gran cantidad de lo que puede llamarse buenas prácticas, también emergieron dificultades que se ubicaron en la categoría de retos. Las categorías definitivas fueron:

a) Éxito escolar

b) Logros en el trabajo colaborativo para apoyar el éxito escolar

c) Retos ante la promoción conjunta del éxito escolar

\section{Hallazgos}

A continuación se definen las categorías y se explican con las citas textuales de las personas participantes.

\section{1. Éxito escolar}

Las personas participantes entienden el éxito escolar como el desarrollo de habilidades que prepara a estudiantes de primaria para la vida y que permite la aplicación de lo aprendido en la cotidianidad, incluyendo la comprensión de las consecuencias de las propias decisiones; se refleja en motivaciones que conducen al desarrollo de las personas, a la solución de problemas y al establecimiento de comunicación. Éxito escolar es salir adelante a pesar de las dificultades; es desarrollar herramientas emocionales para el manejo de la frustración y la edificación de la persistencia. El éxito escolar es la vivencia de experiencias que hacen que la persona estudiante de primaria crea en sus capacidades y disfrute de buenas relaciones en el centro educativo.

Algunas de las citas textuales que sustentan la primera parte de esta definición son las siguientes:

[...] solucionar problemas y enfrentarse a los retos, porque la cosa cada día se pone más difícil sobre todo en la parte laboral, ahorita nosotros estamos recibiendo una generación que está superexpuesta a cosas que la generación mía no tenía, los acosos por redes sociales, las drogas, ese montón de cosas (P2).

Para mí es importante que logre un aprendizaje significativo y pueda ponerlo en práctica en su vida diaria (P5).

Todas las personas participantes mencionaron la integralidad como característica principal del éxito escolar. En este sentido, las calificaciones y la aprobación de niveles educativos es un componente junto con otros que se encuentran en la literatura, pero que el grupo de informantes amplía al referirse al desarrollo personal, la construcción de la solidaridad, el saber que se cuenta con apoyos, el sentido de bienestar y las oportunidades para contribuir. De acuerdo con sus aportes, el éxito escolar conduce a la formación de ciudadanas y ciudadanos conscientes, que aportan a la sociedad con satisfacción y, a la vez, criticidad; se manifiesta por medio de la curiosidad y el gusto que sienten las estudiantes y los estudiantes de primaria en sus vivencias en los centros educativos. Algunas de las expresiones en relación con la integralidad del éxito escolar son las siguientes:

[...] un chico o una chica que tenga éxito escolar es un chico o una chica que se sienta pleno en el ambiente educativo, no solo porque sacó buenas calificaciones, sino porque logra tener relaciones interpersonales sanas, bonitas. Que sepa que tiene personas a quien recurrir en un centro educativo; 
que aporta desde lo que recibe en su casa (P15).

[...] que disfruten todo lo que aprendan, que sientan esa curiosidad, ese interés, esas ganas de aprender (P7).

El éxito escolar es un proceso, por lo que las bases del éxito en primaria se edifican desde los niveles iniciales, y las bases del éxito en el colegio, desde la escuela. Desde la visión de la integralidad del éxito escolar, las personas participantes consideran importante el tema de las calificaciones tanto para quienes obtienen bajos puntajes como para estudiantes de primaria que tienen un alto rendimiento:

[...] es toda la parte en cómo esa persona estudiante se sienta en esta institución. Cómo puede solucionar las situaciones, cómo son sus habilidades sociales, cómo es su comunicación. Obviamente se enfoca también en las notas (P16).

[...] que no es solamente enseñarles a leer, es que analicen lo que lean, que comprendan lo que lean [...] a decir lo que sienten, no callarse (P9).

La Figura 1 es una representación de las habilidades cuyo desarrollo refleja éxito escolar de las estudiantes y los estudiantes de primaria.

La perspectiva de las personas participantes con respecto al éxito escolar como proceso, cuya característica central es la integralidad, puede verse en la
Con respecto a las formas de edificar el éxito escolar, de acuerdo con las personas informantes, este se construye proporcionando oportunidades para que las niñas y los niños se sientan capaces, perciban el interés en sus procesos por parte de las personas involucradas y no sientan temor. Para construir el éxito se requiere conocer a cada estudiante, su contexto familiar y realidades, para así tener respuestas pertinentes a sus necesidades y "tratar de adaptarnos un poco más a ellos, y no ellos a nosotros" (P12). Debido a que se concibe el éxito como un fenómeno integral, se deben fortalecer el manejo de emociones y la autorregulación a la vez que se trabaja lo académico, por ejemplo, "de Matemáticas o de Espańol, en los cuales se involucra también la parte emocional y conductual [...] que les permita sentirse que se enamoran de escribir, de leer, de las Matemáticas, y que les sea también algo placentero" (P4).

Otros aportes que se refieren a las formas de construir éxito escolar de estudiantes de primaria son los siguientes:

Una profesora que me marcó mucho, por su forma tan asertiva, tan tierna también, de trabajar, de acompańarnos a los chicos y chicas que estábamos en el grupo [...] tenemos que hacer una contrapropuesta a lo que a veces creen [...] no podemos seguir con patrones de exhibir en público a chicos o chicas cuando hacen algo inadecuado (P3).

\section{Figura 1. Habilidades que reflejan éxito escolar}

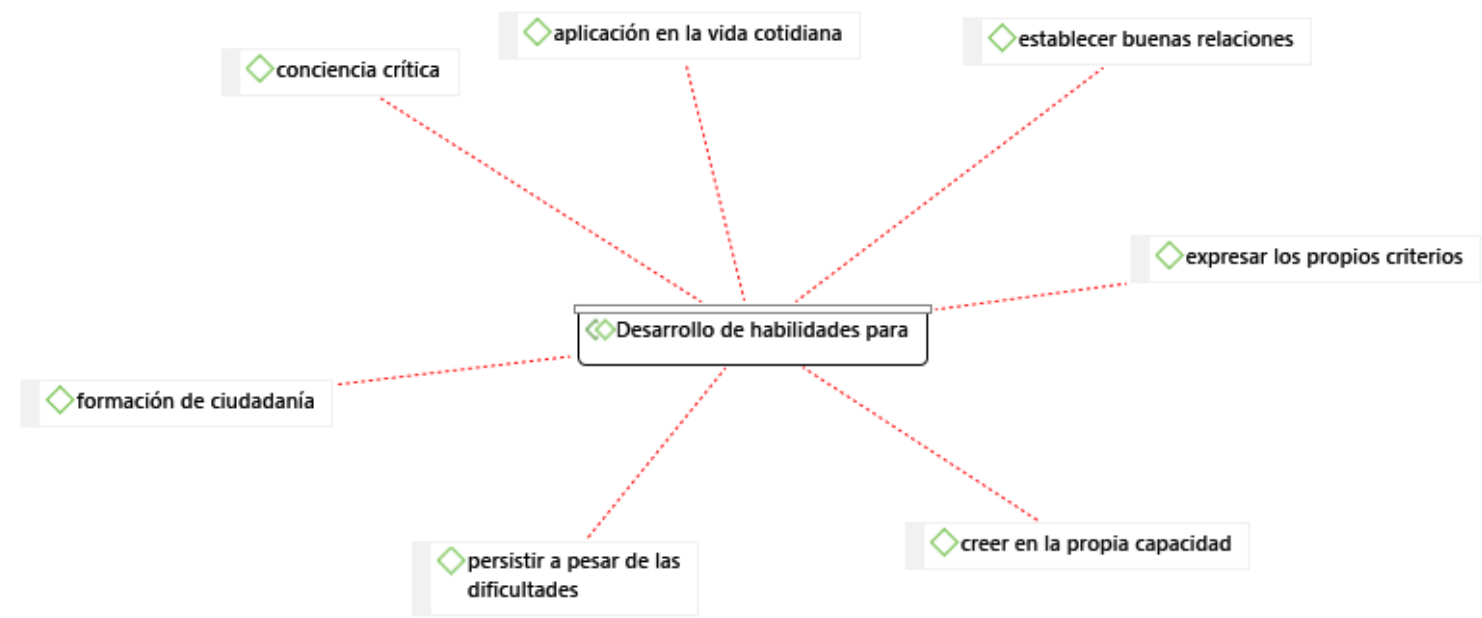

Nota: Elaboración propia con base en aportes de participantes. 


\section{Figura 2. Integralidad del proceso de éxito escolar}

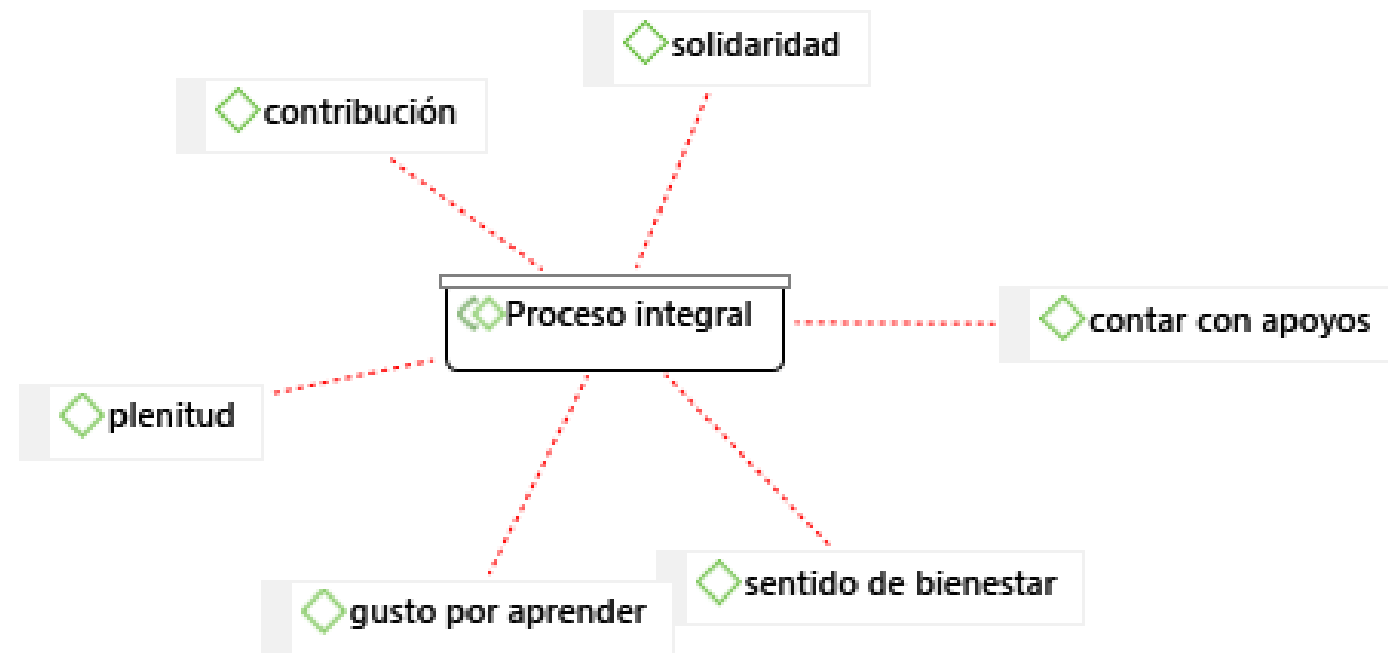

Nota: Elaboración propia con base en aportes de participantes.

Yo también les doy la confianza para que puedan preguntar y preguntar, no importa diez, once veces, lo que no entendieron para volverlo a retomar, hasta que lo entiendan (P13).

Asimismo, se requiere el acompañamiento de la familia y de la totalidad de la institución. Otro elemento para la construcción del éxito de estudiantes de primaria es la provisión de ambientes que reconozcan los aportes del estudiantado y en los que se den múltiples muestras de solidaridad. La labor interdisciplinaria de promoción del éxito escolar implica respetar el ritmo de aprendizaje de cada estudiante y trabajar de forma colaborativa; esta colaboración debe extenderse hacia lo artístico y lo deportivo, ya que contribuye con el éxito integral y el bienestar de las niñas y los niños. Tal y como lo plantea una participante, "es un trabajo interdisciplinario, un trabajo donde la maestra interviene, donde los servicios de apoyo intervienen, donde orientación interviene, o sea, donde todos intervenimos" (P14). Otra persona agrega:

[...] ese seguimiento que nosotros damos, para mí es lo más valioso, o sea, no creo que haya orientadora que podría hacerlo sola, ni educadora especial que podría hacerlo sola, ni docente que podría hacerlo sola (P14).
Con respecto a la implicación de todo el personal en función del bienestar de estudiantes de primaria, una participante indica que:

[...] en la guarda, en las cocineras, eh, las personas conserjes, la oficinista, o sea, todos hablamos el mismo idioma, o sea, aquí estamos todos por los estudiantes (P16).

Algunas de las personas participantes manifiestan que consideran que las familias son integrantes de los equipos colaborativos:

[...] las mismas familias también se han ido encargando de decirnos a nosotros "de ustedes ocupamos [necesitamos] esto". Y ahí es donde también se da un trabajo colaborativo, ¿verdad? (P15).

Es importante acotar que para lograr todo lo anterior se requieren ambientes propicios, abiertos a iniciativas que favorezcan al estudiantado, al personal y a las familias, "un ambiente agradable donde el docente diga 'Qué lindo, voy a ir a trabajar', y no 'Qué pereza', ¿verdad?, donde si hay algún inconveniente entre dos o más compañeros, acercarse y de una vez solucionarlo para que no se vaya a hacer más grande el problema” (P1). Esta misma participante enfatiza en la solidaridad:

[...] no logramos avanzar si no hay una solidaridad con el estudiante, o con el docente compañero, 
para poder llevar a cabo todos esos objetivos [...] que yo me ponga en el lugar del otro, cómo se siente, cuáles son sus necesidades para lograr que el éxito sea el que nosotros deseamos (P1).

En la Figura 3 se ilustran los elementos con los que se construye el proceso de éxito escolar de estudiantes de primaria, de acuerdo con las personas participantes.

\subsection{Logros en el trabajo colaborativo para apoyar el éxito escolar}

Para poder profundizar en el análisis del trabajo colaborativo, se indagó acerca de las acciones interdisciplinarias que implementan las personas participantes. Con base en lo que expresaron, se definen estas acciones como las medidas que toman en conjunto, que implican validar los aportes de cada persona integrante del equipo, en función del logro de objetivos comunes, de generar sensibilidad ante la diversidad y de la provisión de condiciones lo más integrales posible para el desarrollo de estudiantes de primaria y la prevención de la exclusión educativa. Son acciones hechas en conjunto que dan mejor resultado que cuando se llevan a cabo de forma aislada por una sola persona. En palabras de las personas participantes:
[...] al observarse, visualizarse un problema desde diferentes perspectivas se abre una amplia gama de estrategias (P6).

Las acciones colaborativas contribuyen con todos los ámbitos del desarrollo, sin descuidar unos al enfatizar en otros. También son una vía para concretar una de las formas de favorecer el éxito escolar, que es demostrarle al estudiante que hay personas interesadas en sus procesos educativos. P5 aporta que considera esencial

El apoyo, que se sientan acompañados en todo momento, conocer al estudiante de forma integral, más allá de un número, de una calificación [...] es que hay que ver por qué llega tarde, por qué no viene, por qué se duerme.

Las acciones colaborativas comienzan con la puesta en común del conocimiento de la población para identificar sus fortalezas y necesidades, y continúan con el monitoreo y seguimiento. Implican apoyo y enriquecimiento mutuo de las personas participantes. No son eventos aislados, sino un proceso permanente de construcción que permite definir en conjunto estrategias de mediación y determinar si las intervenciones se realizan de forma individual, en pequeños grupos o con todo un nivel. Tal y como lo expone una participante:

\section{Figura 3. Elementos para la construcción del éxito escolar}

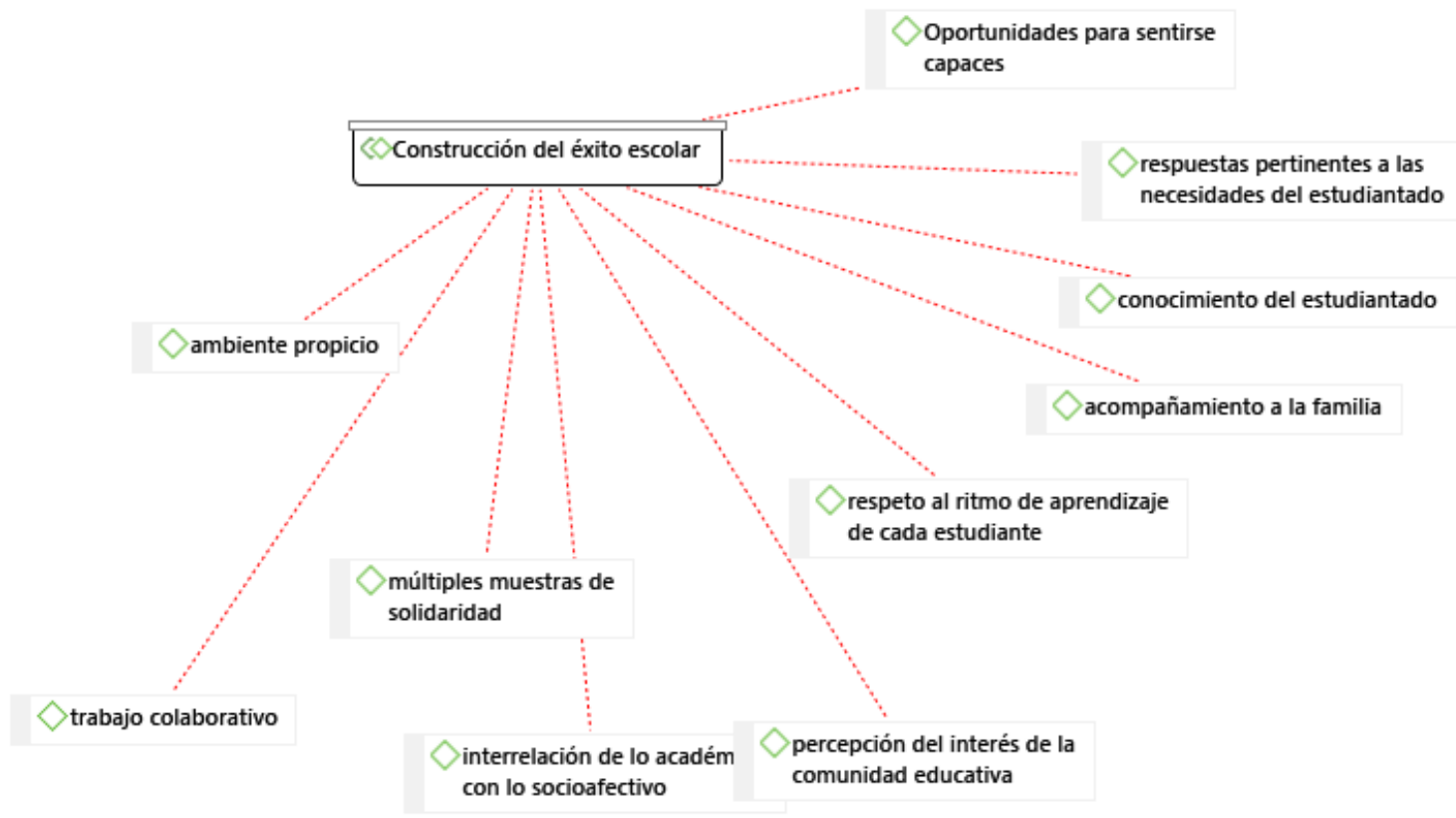

Nota: Elaboración propia con base en aportes de participantes. 
[...] analizar esa realidad de cada grupo, de cada aula, porque cada uno es diferente y a partir de ahí se determinan las necesidades y las situaciones. Entonces, primero conversamos de esas situaciones que hemos visto y como equipo pensamos en qué experiencias o situaciones de mediación deberíamos promover con los chicos, algunas las trabajamos de manera individual cuando vamos a trabajar en el aula, pero muchas las planificamos en talleres (P4).

Otras expresiones ilustran la forma en que las personas participantes conciben las acciones colaborativas, por ejemplo:

[...] con otra visión, entonces viene como a favorecer eso, es decir, yo conozco muy bien a mis estudiantes, pero este apoyo que me está dando la compañera, ¿verdad?, es un acompañamiento necesario (P6).

El enriquecimiento que significa la interdisciplinariedad puede observarse en el comentario de una participante en relación con el agrado que produce en las niñas y los niños la presencia de docentes de Educación Especial y profesionales de Orientación en las aulas:

[...] están supercontentos de que las profes lleguen [...] saben que van a hacer cosas diferentes, ¿verdad?, entonces vieras que las esperan cuando yo digo: viene la profe $\mathrm{X}$, viene la profe $\mathrm{Y}$, o viene la profe $Z$ [...] bueno, se motivan (P12).

Las personas participantes informan acerca de la efectividad del trabajo colaborativo para el logro del involucramiento de las familias; la efectividad también la han observado en su desarrollo profesional:

[...] hacer una intervención en un equipo ha sido más, más este, más valioso, no, no, valioso no es la palabra, ha sido más efectivo que hacer intervenciones por separado [...] ese es un espacio donde nosotros estamos logrando interdisciplinariamente que los papás puedan tener mayor compromiso y que eso repercuta en un éxito escolar (P14).

[...] yo creo que los docentes necesitamos estar aprendiendo, y qué mejor que aprender de la experiencia del otro y de las estrategias exitosas que han tenido los otros (P4).
Las acciones colaborativas trascienden las disciplinas consideradas en la presente investigación hacia todas las otras personas integrantes de la comunidad educati$\mathrm{va}$, tales como docentes de materias complementarias, e incluso hacia otras instituciones, ya que esto favorece el bienestar y el éxito de sus estudiantes de primaria:

[...] hay situaciones de estrategias en las cuales ellas ya son especialistas, las de Preescolar, las de Primer y Segundo ciclo, que cada quien tiene su especialidad, igual es con los compañeros de Inglés, de Música o cualquiera, cada quién tiene su especialidad (P16).

[Acerca de chat con colegas de otras instituciones]: [...] porque como en Primaria solo es un orientador, tenemos un chat para apoyar (P17).

Las familias también son consideradas integrantes de los equipos colaborativos:

[...] se citan padres de familia en conjunto con los servicios de apoyo y la docente guía, entonces yo creo que, bueno, ese trabajo en equipo hace que se pueda dar el éxito escolar (P11).

A pesar de que las personas entrevistadas se refieren a la relevancia de que se involucre toda la comunidad educativa en las acciones en pro del logro y del bienestar de estudiantes de primaria, en la práctica cotidiana son más frecuentes las reuniones que se llevan a cabo de forma bilateral, es decir, de dos personas; aparte del apoyo desde las diferentes disciplinas, una práctica común es que como colegas se hacen presentes con el fin de brindar acompañamiento cuando se han programado reuniones con familiares o con la dirección de la escuela. Son menos frecuentes las oportunidades en que se reúnen juntas las disciplinas de Orientación, Educación Especial y Educción General Básica, lo hacen en relación con las situaciones más complejas que atienden. Asimismo, se hacen en conjunto las referencias a instituciones externas como los Equipos Básicos de Atención Integral en Salud (EBAIS), clínicas o el Hospital Nacional de Nińos.

En la Figura 4 se presentan las principales acciones que las personas participantes llevan a cabo de forma colaborativa para promover el éxito escolar de estudiantes de primaria a su cargo. 


\section{Figura 4. Acciones colaborativas}

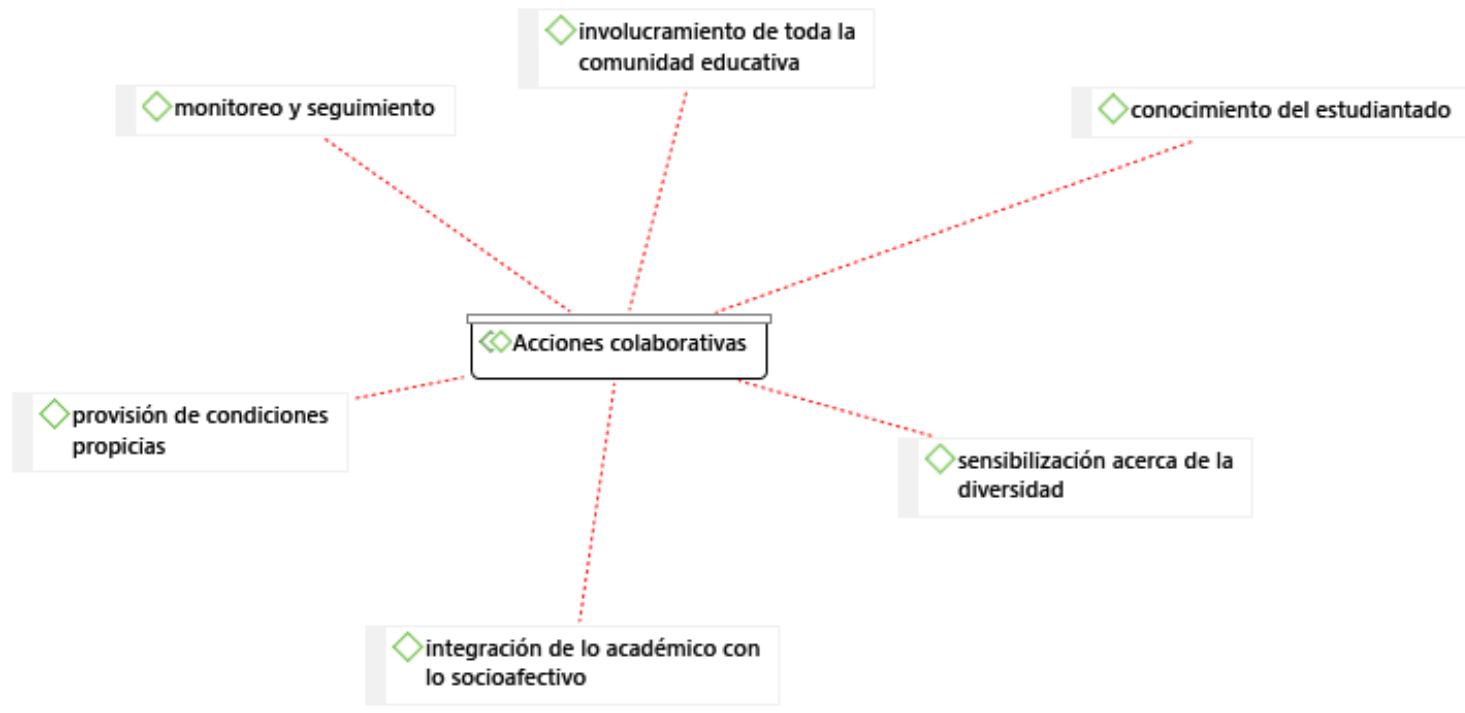

Nota: Elaboración propia con base en aportes de participantes.

Además de describir las acciones colaborativas, las personas participantes se refirieron a las formas de edificarlas y a lo que se requiere para que funcionen constructivamente. Dentro de los requerimientos se encuentran la empatía, el respeto y la valoración de los aportes de cada persona. Algunas de sus convicciones son las siguientes:

[...] siento que no solo las ideas mías valen, valen las de todos (P1).

[...] ellas y yo conversamos y yo pregunto o ellas me recomiendan: "puede modificar tal cosa", pero nunca, nunca, como imponiendo (P9).

[...] saque un tiempo, reúnase con ellas, escúchelas, qué es lo que necesitan, enséñeles cómo es que usted trabaja (P14).

[...] me siento pero orgullosísima del equipo, porque son demasiado profesionales, cada una toma tan en serio y con tanta vocación y con tanta entrega su trabajo, que estoy realizada con las compañeras que tengo (P7).

La escucha también se ha aplicado fuera del centro educativo, ya que se hacen consultas a otras instituciones con respecto a servicios que no están disponibles en su escuela o a la implementación de las líneas de acción:
[...] fue muy rico porque escuchamos la propuesta de otros centros educativos y es muy interesante, ¿verdad?, porque algunos tal vez se enfocaron en unas cosas y nosotras en otras, pero al ver los diferentes enfoques uno podría decir ahí deberíamos tomar lo mejor de cada uno y pensar qué se ajusta también a este centro educativo (P4).

No menos importante es reconocer la importancia del trabajo colaborativo y de prepararse para llevarlo a cabo. Con base en su experiencia, P6 recomienda:

[...] primero, motivar a las maestras; segundo, que el plan que se está estableciendo tenga un objetivo claro; tercero, para mí es superimportante que realmente exista una coordinación, porque si no existe las maestras somos como islas [...] implica que tengamos tiempo, una lección a la semana, dos lecciones a la semana, para poder socializar ese trabajo que se va a hacer con las maestras de Educación Especial, de Orientación.

Para llevar a cabo de forma efectiva el trabajo colaborativo, las personas entrevistadas y otro personal de las escuelas participantes han hecho uso de aprendizajes de experiencias anteriores, tales como la organización de festivales, ferias, conmemoraciones, "recreos felices" y otros proyectos institucionales. Una persona participante lo ejemplificó así: 
[...] todos trabajamos: festival de Inglés, feria científica, festival de las artes, todos aportamos un granito de arena en lo que sabemos (P10).

Cabe agregar que los elementos que edifican el trabajo colaborativo guardan gran similitud con los que promueven la educación inclusiva, entre los que se destacan un ambiente de trabajo agradable, en el que se da valor a las ideas de todas las personas, el gusto por el trabajo y por las acciones que se hacen en conjunto, la escucha respetuosa, la creatividad para adecuarse a las limitaciones y el trato cordial que transmite que no estamos solas o solos. Esto coincide con lo planteado por Barrow y Mamlin (2016) en relación con los grupos de trabajo colaborativo interdisciplinario. P8 expresó con entusiasmo que:

[...] me gusta, a mí me gusta lo que hago, me gusta lo que hago, trabajar con $\mathrm{X}$.

Con una mayor presencia en las aulas de las personas integrantes de equipos, es esencial que ninguna parte se sienta fiscalizada, sino que la forma de trabajar sea muy respetuosa y en función de la satisfacción de las necesidades de estudiantes de primaria.

Que docentes de aula no crean que la presencia de Educación Especial es de fiscalización, es de trabajo en equipo (P3).

[...] como nosotros estamos en las aulas, detectamos muchas situaciones que a veces al docente por el asunto de estar con muchos nińos se les pueden escapar (P4).

Las formas en que las personas participantes han edificado el trabajo colaborativo en sus instituciones se muestran en la Figura 5.

\subsection{Retos ante la promoción conjunta del éxito escolar}

El hecho de que se lleven a cabo múltiples acciones colaborativas que promuevan el éxito escolar de estudiantes de primaria no significa que no existan retos que dificultan la implementación de tales acciones. Si bien se hacen múltiples esfuerzos por evitar o compensar dificultades, los siguientes cinco aspectos representan retos o desafíos, de acuerdo con las personas participantes:

a) Necesidad de contar con espacios y mayor cantidad de tiempo para el planeamiento y la implementación del trabajo colaborativo, así como la socialización del conocimiento entre las personas de los equipos. La coordinación entre todo el equipo se lleva a cabo para la atención de casos más complejos. Como lo expone P15, tratan de "andar en la misma sintonía", pero sienten constantemente la insuficiencia de tiempo disponible, lo que también afecta la consistencia en el seguimiento a los planes establecidos en conjunto. Las siguientes expresiones son un reflejo de este desafío:

\section{Figura 5. Edificación del trabajo colaborativo}

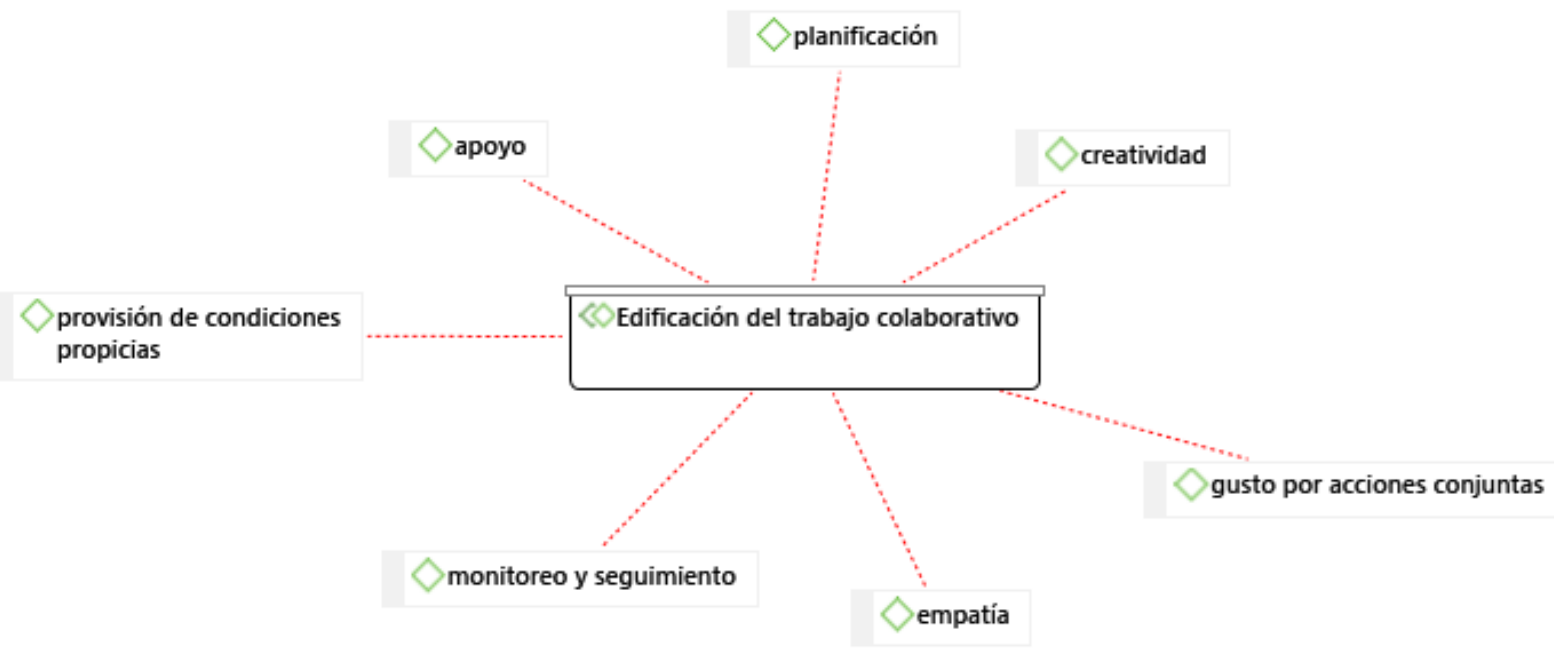

Nota: Elaboración propia con base en aportes de participantes. 
Sí, sinceramente siento que nos falta ese espacio, para ir juntas las tres (P17).

[...] si son casos muy serios sí lo atendemos todas, ¿̇verdad?, eh, las dos compañeras, tanto la de Apoyo como la de Emocionales y yo, y la orientadora inclusive (P12).

b) Efectos en las niñas y los niños de privaciones, problemáticas sociales y un acompañamiento inestable por parte de las familias. Las personas participantes hacen múltiples esfuerzos por aprovechar los aportes de madres, padres y personas encargadas, pero se sienten retadas:

[...] están pidiendo a gritos, pues cómo lo hago, cómo lo hago para no lastimar, cómo lo hago inclusive para desaprender y aprender a hacerlo ahora con mis hijos (P15).

[...] violencia, negligencia, ausentismo montones [...] eh, poco apoyo en el hogar; tenemos limitaciones de padres que no saben leer y escribir (P17).

c) Incremento de la participación en acciones colaborativas de personas encargadas de materias y actividades artísticas y deportivas.

[...] hacer manejo en el aula, pero las complementarias no, entonces hicimos un documento de recomendaciones para los profesores de materias complementarias (P17).

d) Disponibilidad de recursos para atender la diversidad de todos los grupos. Es decir, se requieren más recursos humanos para atender la amplia gama de características y necesidades y ejecutar medidas preventivas que beneficien a la totalidad de estudiantes de primaria. Una participante manifestó su preocupación en relación con la escasa variedad de adecuaciones que se aplican en las aulas; otras consideran en ocasiones que sigue siendo necesario el trabajo individual:

¿Cuáles son las adecuaciones que se aplican en las aulas? Darle más tiempo al estudiante, ponerlo adelante [...] más tiempo en los exámenes, fórmulas, de ahí no salimos [...] estamos llegando solo a esos cuatro aspectos (P12).

[...] porque hay ciertos estudiantes que lo requerimos de manera individual, porque en grupo completo no es tan fácil darle esa atención específica (P4).

e) Mayor dedicación a la prevención que a la solución de problemas. Debido a la presencia de problemáticas complejas que demandan bastante dedicación, se presenta el riesgo de dejar sin atender tempranamente algunas necesidades "más leves", lo que podría perjudicar al estudiantado de primaria que cuenta con mayores ventajas, pero incluso así presentan necesidades. $\mathrm{Al}$ respecto, las personas participantes indican que

[...] de alguna manera, jerarquizar los casos nos ayuda a nosotros a mantener el foco de atención en los que necesitan más (P14).

[...] el resto de sus áreas, digamos, que tiene ambientes nutricios para estar, pero para los que no, es donde es vital el trabajo interdisciplinario (P15).

Los retos a los que se enfrentan las personas informantes coinciden con lo que expone Bartolomé (2017) en cuanto el desarrollo integral del estudiantado implica afrontar múltiples condiciones, las cuales están más acentuadas en poblaciones desfavorecidas.

\section{Conclusiones}

Los aportes de las personas participantes enriquecen los conocimientos disponibles acerca de las temáticas tratadas. El trabajo colaborativo es uno de los componentes principales de la educación inclusiva, y facilita un abordaje que toma en cuenta los múltiples factores que intervienen en el éxito escolar de la totalidad de estudiantes de primaria. Las personas participantes en la investigación lo edifican en su quehacer cotidiano con esfuerzo, constancia y respeto mutuo. Al igual que la educación inclusiva y el éxito escolar, el trabajo colaborativo interdisciplinario es visto como un proceso.

Este grupo de profesionales de la Educación concibe el éxito escolar de una manera integral y procesual. Desde sus puntos de vista, el éxito escolar de estudiantes de primaria se constata en la puesta en práctica de los aprendizajes en la vida cotidiana, para el bienestar en todos los ámbitos. Consiste en el desarrollo de 
habilidades que se aplican de forma interrelacionada en lo afectivo, lo interpersonal y lo académico. Cuando las niñas y los niños son persistentes, creen en sus capacidades, pueden solucionar problemas y se sienten parte de la comunidad educativa, se puede decir que han alcanzado el éxito escolar. También son manifestaciones de éxito la curiosidad, el gusto por aprender y la persistencia. Las personas participantes coinciden en que el proceso de éxito escolar se construye en ambientes caracterizados por la aceptación, la implementación conjunta de soluciones y el apoyo para enfrentar retos.

Además, el éxito escolar como proceso se construye con la participación de múltiples actores que transmiten interés, manifiestan solidaridad y brindan oportunidades para que las niñas y los niños identifiquen y apliquen sus capacidades. Las familias son actores centrales que apoyan el proceso y a la vez requieren el acompañamiento del personal de las escuelas. El éxito escolar tiene alta relevancia social, porque su fin último es la formación de una ciudadanía consciente, crítica y que siente satisfacción al aportar.

Las personas participantes representantes de profesionales en Orientación, docentes de Educación Especial, profesorado de Educación General Básica de primer y segundo ciclo y de Administración Educativa resaltan la importancia de un clima de trabajo agradable, de la valoración de los aportes de todas las personas, del disfrute de las acciones que llevan a cabo de forma colaborativa, de las relaciones respetuosas y de la creatividad para enfrentar los múltiples desafíos que cada día enfrentan en su trabajo como educadores de primaria. Al igual que el éxito escolar, consideran que el trabajo colaborativo es un proceso; cabe reiterar que la educación inclusiva también constituye un proceso (Schuelka, 2018), por lo que cobra relevancia su constante construcción en los centros educativos de primaria en general y en los grupos interdisciplinarios en particular.

Se reiteró que una de las principales dificultades que enfrentan los equipos colaborativos entrevistados es la insuficiencia de tiempo para trabajar en conjunto en el planeamiento y sistematización del trabajo conjunto. También implica un desafío permanente disminuir los efectos en las niñas y los niños en condiciones de pobreza, riesgos psicosociales e inconsistencia por parte de algunas familias.

Otra tarea a la que dedican esfuerzos es la implicación de toda la comunidad educativa en las acciones para llevar a la práctica la inclusión auténtica de la totalidad de estudiantes de primaria. Además, para atender de forma lo más integral posible la diversidad y necesidades del alumnado, todas las personas participantes resaltan la pertinencia de contar con mayor cantidad de personal.

Por último, pero no menos importante, está el tema de la pertinencia de aumentar el trabajo colaborativo para la solución de problemas, para enfatizar en las medidas preventivas integrales, centradas en la motivación, la oportunidad y el sentido de valía del estudiantado de primaria.

\section{Agradecimientos y reconocimientos}

Las autoras agradecen la apertura de las instituciones y los aportes de cada una de las personas participantes, los cuales van a enriquecer las vivencias de trabajo colaborativo en otras escuelas primarias. Asimismo, el trabajo dedicado y responsable de las asistentes Lindsay Fallas Masís, de la carrera de Educación Especial, y Natalia Cordero Miranda, de la carrera de Orientación.

El proyecto contó con el apoyo de la Escuela de Orientación y Educación Especial y el Instituto de Investigación en Educación (INIE), ambos de la Facultad de Educación, Universidad de Costa Rica; fue financiado por la Vicerrectoría de Investigación de esta institución (Proyecto $\mathrm{N}^{\circ}$ 724-B0-076).

\section{Referencias bibliográficas}

Alba, C., Sánchez, J., \& Zubillaga, A. (2011). Diseño Universal para el Aprendizaje (DUA). Pautas para su introducción en el currículo. España: Dualetic. https://cutt.ly/6jDfak6 
Álvarez-Gayou, J. (2003). Cómo hacer investigación cualitativa. Fundamentos y metodología.

https://cutt.ly/UjDfjVS

Aristimuño, A., \& Parodi, J. (2017). Un caso real de combate al fracaso en la educación pública: una cuestión de acompañamiento, liderazgo y cultura organizacional. REICE, 15(4), 141-157.

https://doi.org/10.15366/reice2017.15.4.008

Arnaiz, P. (2012). Escuelas eficaces e inclusivas: cómo favorecer su desarrollo. Educatio Siglo XXI, 30(1), 25-44. https://cutt.ly/ljDfAuH

Arnaiz, P., De Haro, R., \& Azorín, C. M. (2018). Redes de apoyo y colaboración para la mejora de la educación inclusiva. Revista de Curriculum y Formación del Profesorado, 22(2), 29-49. https://syr.us/I1C

Barrow, J., \& Mamlin, N. (2016). Collaboration Between Professional School Counselors and Special Education Teachers. ACA Knowledge Center. American Counseling Association. https://cutt.ly/LjDfHBH

Bartolomé, M. (2017). Diversidad educativa ¿Un potencial desconocido? Revista de Investigación Educativa, 35(1), 15-33.

https://doi.org/10.6018/rie.35.1.275031

Bertoldi, S., \& Enrico, L. (2012). La intervención (inter)disciplinar de los equipos técnicos en educación: alcances y límites. Revista Ciencia, Docencia y Tecnologia, (23)45, 131-146. https://cutt.ly/ZjDjklS

Bowen, G. (2008). Naturalistic inquiry and the saturation concept: a research note. Qualitative Research, $8(1), 137-152$.

https://doi.org/10.1177/1468794107085301

Buckley, M. \& Mahdavi, J. (2018). Bringing children from the margins to the page: School counselors supporting students with learning disabilities. Sonoma State University. Journal of School Counseling, 16(23). https://cutt.ly/5jDjRst

Castillo, N. (2020). Fenomenología como método de investigación cualitativa: preguntas desde la práctica investigativa. Revista Latinoamericana de
Metodología de la Investigación Social ReLMIS, 20, 7-18. https://cutt.ly/UjDvJUP

Confederación Española de Asociaciones de Padres y Madres de Alumnos. (2011). El éxito escolar. ¿Cómo pueden contribuir las familias al alumnado?

https://cutt.ly/PjDj9av

Coronado-Hijón, A. (2018). Resiliencia escolar: la superación de barreras ante el aprendizaje y la participación. https://cutt.ly/rjDko4L

Gill M.J. (2020). Phenomenological approaches to research. En N. \& M. Järvinen (Eds.), Qualitative Analysis: Eight approaches (pp. 73-94). Londres: Sage. https://n9.cl/e2t5s

MacQueen, K. (2007). Enriquecimiento de los hechos y las cifras. En A. Álvarez (Comp.), Investigación cualitativa (pp. 9-10). La Habana: Editorial Ciencias Médicas.

Marshak, L., Dandeneau, C., Prezant, F., \& L'Amoreaux, N. (2010). The School Counselor's Guide to Helping Students with Disabilities. San Francisco, California: Jossey-Bass Teacher.

Ministerio de Educación Pública. (2013). Lineamientos sobre apoyos curriculares y de acceso para la atención de necesidades educativas del estudiantado en la Educación General Básica y Educación Diversificada. https://cutt.ly/mjDkgzD

Ministerio de Educación Pública. (2017a). Orientaciones técnico-administrativas para operacionalizar el Servicio de Orientación a nivel regional y centro educativo, 2017. Departamento de Orientación Educativa y Vocacional. https://cutt.ly/FjDkxWM

Ministerio de Educación Pública. (2017b). Educar para una Nueva Ciudadanía. Programas de Estudio de Orientación Primero, Segundo y Tercer Ciclos de la Educación General Básica y Educación Diversificada. San José, Costa Rica. https://cutt.ly/fjDkmRb

Ministerio de Educación Pública. (2018). Hacia una educación inclusiva. Lineas de acción para los servicios de apoyo educativo que se brindan desde la Educación 
Especial en la Educación Preescolar y en Primero y Segundo Ciclos de la Educación General Básica. San José, Costa Rica. https://cutt.ly/sjDkRXb

Mondino, V. (2017). La experiencia de un trabajo en un equipo interdisciplinario en el ámbito educativo. https://cutt.ly/4jDkJgg

Nelsen, J., Lott, L., \& Glenn, S. (2016). Disciplina positiva en el salón de clase. México: Editorial Rondine.

Neto, A. S., Ávila, E. G., Sales, T. R., Amorim, S. S., Nunes. A. F., \& Santos, V. M. (2018). Educação inclusiva: uma escola para todos. Revista Educação Especial, 31(60), 81-92.

http://dx.doi.org/10.5902/1984686X24091
Programa Estado de la Nación en Desarrollo Humano Sostenible. (2017). Sexto informe del estado de la educación costarricense. San José, Costa Rica: PEN. https://cutt.ly/jjDkZXu

Schuelka, M. J. (2018). Implementing inclusive education. K4D Helpdesk Report. Brighton, UK: Institute of Development Studies. https://syr.us/wll

Taylor, J. (1989). Correcting without criticizing. The encouraging way to talk to children about their misbehavior. Doylestown, PA, EE.UU.: mar*co products, inc.

Ulloa, G. (2017). Psicología de la motivación. San José, Costa Rica, EUNED. 


\section{Anexo 1}

Guía de entrevista

Nombre de la persona entrevistada:

Institución:

Puesto: Fecha de entrevista:

Datos generales:

$\rightarrow$ Tiempo de experiencia laboral

$\rightarrow$ Tiempo de laborar en esta institución

$\rightarrow$ Nivel/es que imparte

$\rightarrow$ Especialidad

Preguntas relacionadas con el objetivo 1 de la investigación: Identificar las acciones colaborativas que realizan las personas profesionales de Orientación, I y II Ciclos de Educación General Básica y de Educación Especial para la promoción del éxito escolar en el estudiantado de escuelas primarias.

Acciones colaborativas se refiere a: actividades, coordinación, procedimientos y seguimiento que se hacen en conjunto.

1. Para usted, ¿qué es éxito escolar?

2. ¿Cuáles acciones y actividades realizan de manera colaborativa las personas profesionales en Orientación, Educación General Básica y Educación Especial, para la promoción del éxito escolar?

3. ¿Cuáles otras personas están involucradas en la promoción del éxito escolar en esta escuela? Describa las acciones colaborativas que realiza con esas otras personas.

4. ¿Cuál es el procedimiento para la atención de estudiantes con riesgo de fracaso escolar (problemas de rendimiento, conducta, "desinterés" por el trabajo en el aula, ausentismo, entre otros) que tienen en esta institución?

5. ¿Qué tipo de seguimiento recomiendan dar a las acciones interdisciplinarias y de trabajo colaborativo?

Preguntas relacionadas con el objetivo 2 de la investigación: Determinar el aporte de profesionales de Orientación, profesorado de I y II Ciclos de Educación General Básica y docentes de Educación Especial, a la promoción del éxito escolar del estudiantado de primaria. 
6. ¿Cuál ha sido el aporte de su disciplina en la promoción del éxito escolar?

7. ¿Cuál es la importancia para el estudiantado de realizar el trabajo colaborativo de las tres disciplinas (Orientación, Educación Especial y Educación General Básica) en la promoción del éxito escolar?

8. ¿Cuál es su opinión acerca de la viabilidad de aplicar los siguientes lemas relacionados con un manejo de la disciplina que favorece la motivación?

"Para que el niño/la niña se comporte bien, no es necesario hacerle sentir mal".

"Es más efectivo el reconocimiento del comportamiento positivo, que la corrección del comportamiento negativo".

"La relación docente-estudiante debe caracterizarse por el respeto mutuo".

\section{CÓMO CITAR:}

Arguedas-Negrini, I., \& Carpio-Brenes, M. A. (2021). Trabajo colaborativo interdisciplinario para la promoción de la educación inclusiva y el éxito escolar de estudiantes de primaria en Costa Rica. RECIE. Revista Caribeña de Investigación Educativa, 5(2), 124-143. https://doi.org/10.32541/recie.2021.v5i2.pp124-143 